\title{
CHANNEL CODING STRATEGIES FOR CELLULAR RADIO
}

Gregory J. Pottie ${ }^{1}$

Electrical Engineering Dept.

University of California, Los Angeles

405 Hilgard Ave.

Los Angeles, CA 90024
A. Robert Calderbank

Mathematical Sciences Research Center

AT\&T Bell Laboratories

600 Mountain Ave.

Murray Hill, NJ 07974

\begin{abstract}
To improve re-use of time/frequency slots in a cellular radio system, it is desirable for the average interference levels seen by all users to be made approximately equal. We provide constructions based on orthogonal latin squares that guarantee different sets of users will interfere in successive slots. We illustrate how this may be combined with convolutional coding to provide large performance improvement with low decoding delay in a slow hopped system.
\end{abstract}

\section{Introduction}

In mobile cellular radio, the dominant impairments are multipath fading and interference from other mobiles. In a conventional time division multiple access system (TDMA), mobiles are assigned slots which they keep from frame to frame, with the interfering mobiles assigned slots in the same way. As interference levels vary widely between slots due to such factors as shadowing and geographic proximity, some mobiles suffer from persistently poor signal to interference ratios (which we shall refer to as C/I). Systems are generally designed for $90 \%$ and $99 \%$ worst case conditions. Therefore, the result of this uneven interference distribution is overly conservative restrictions on frequency re-use between cells, and thus reduced capacity.

If instead slot assignments were arranged such that different interferers were encountered in successive frames or slots and repetition or channel coding used to provide averaging, then the worst case error statistics would improve. In [1] random hopping over eight frequencies was used to average over different interferers and channel conditions. As a result, all frequencies could be re-used between cells. In [2], a combination of coding, explicit frequency re-use restrictions, and slow frequency hopping was employed. Both yielded capacities similar to that of the direct sequence spread spectrum code division multiple access (CDMA) system analyzed in [3]. Here we provide a specific construction of the hopping patterns which when combined with convolutional coding leads to improved performance, without restrictions on frequency re-use between

1. This work supported in part by AT\&T Bell Laboratories and ARPA contract DAAB07-93-C-C501. 
cells. Performance for our slow-hopped example is similar to that obtained in [4], but with lesser complexity. In that system, long rate-1/2 convolutional codes and hopping patterns with low cross-correlation were employed. In section II we describe our model of the propagation environment and discuss the need for randomization of interferers in TDMA or hybrid frequency hopped TDMA. In section III we describe our construction, which is based on orthogonal latin squares. To achieve any benefits with this technique, channel coding must be used. We discuss the basic coding tradeoffs in section IV, and illustrate them with two related computer simulated examples. We demonstrate significant gain with low delay (20-40 ms) and modest complexity. In section V we present our conclusions.

\section{The interference environment}

We follow the simplified propagation model presented in [3], and focus on the reverse or uplink of mobile to base station. This choice is arbitrary for synchronous systems, but assists in comparison of results in the literature. Mobiles are assumed to be uniformly distributed on an infinite plane, with base stations arranged in a uniform hexagonal pattern. Power drops with the fourth power of distance. In addition, there is independent log-normal shadowing for each track. The received power level for a mobile $m$ at distance $d$ from a base station is then

$$
\mathrm{P}=\frac{10^{0.1 \xi} \mathrm{P}_{\mathrm{m}}}{\mathrm{d}^{4}}
$$

where $\mathrm{P}_{\mathrm{m}}$ is the mobile's transmit power, and $\xi$ is a Gaussian random variable with zero mean and standard deviation $\sigma=8$. The mobiles are assigned to the base station with the minimum propagation loss.

One practical power control strategy is to attempt to make the power level received at the base station approximately the same for all mobiles in the cell. The specific level depends on the margin required against additive white Gaussian noise, but we shall assume the power level to be unity, since all powers and interference levels will scale. Let cell 0 be our reference cell, i.e., the set of mobiles communicating with base station 0 . Then the interference power encountered at the base station of cell 0 from a mobile belonging to cell $\mathrm{i}$ is

$$
I=\left(\frac{r_{i}}{r_{0}}\right)^{4} 10^{0.1\left(\xi_{0}-\xi_{i}\right)}
$$


where

$\mathrm{r}_{\mathrm{i}}=$ distance of mobile to base station $\mathrm{i}$

$\mathrm{r}_{0}=$ distance of mobile to base station 0

$\xi_{0}, \xi_{\mathrm{i}}=$ shadowing random variables for tracks to base stations 0 and i respectively.

Shadowing can be assumed to be constant over the short run. With this propagation model and channel assignment strategy, most of the interference encountered in cell 0 is generated in the two surrounding rings of cells.

Note that shadowing significantly reduces the geographic isolation of cells. Computations performed over the first two complete rings with only fourth power propagation loss and with power controlled for equal received levels at the home base stations indicate that a regular frequency reuse pattern of $1 / 3$ improves $\mathrm{C} / \mathrm{I}$ by close to $13 \mathrm{~dB}$ compared to use of all frequencies in all cells. However, when shadowing is included in the model the improvement shrinks to $7 \mathrm{~dB}$. This compares to a gain of $4.8 \mathrm{~dB}$ which might be obtained just by reducing the density of users in all cells by this same fraction. This may be intuitively explained as follows. With shadowing, the hexagonal cell geometry breaks down and some mobiles may wander far outside these nominal boundaries. In particular, mobiles belonging to the second ring of cells may wander much closer to the base station for cell 0 . At the same time, the interference power due to such mobiles is upper bounded by 1 , since otherwise they would be handed off to cell 0 . With only attenuation with distance, most interference power originated in the region of the first ring of cells near the boundary with cell 0 , with interference power near 1 . Shadowing merely smears out this region. Thus, the probability of large interference power increases signficantly for mobiles assigned to the second ring of cells, while not increasing at all for the first ring of cells. The end result is that it does not matter much for the average $\mathrm{C} / \mathrm{I}$ whether a reduction in mobile density is achieved through explicit frequency re-use patterns or through limits on numbers within each cell, with full frequency re-use. The advantage of the latter approach is that the limits can easily be made adaptive, and no frequency use planning is required.

For a system with frequency re-use of 1 , by symmetry, whether one assumes there to be three perfect $120^{\circ}$ sectors or ordinary cells with $1 / 3$ the density of mobiles the interference levels will be the same. Consequently, all our computations will be performed assuming no sectorization; the only normalization required to directly compare our results to a sectorized system is to multiply the number of mobiles by 3 .

Our simulations assume non-standard narrowband ( $<20 \mathrm{kHz}$ ) systems. Consequently, a rea- 
sonable worst-case model for the short term channel conditions is Rayleigh fading, i.e., the signal is multiplied by a complex Gaussian, with variance in each dimension equal to half the computed long-term power. We suppose that transmission is divided into time or frequency slots. Time slots may hop in position within the frame or in frequency from frame to frame. Usually we assume that fading is independent from slot to slot for any given user, which may be achieved by some combination of delay and frequency agility. We further assume that the slots are short, so that the signal level is constant over the slot (including Rayleigh fading).

In such a system, if mobiles in nearby cells share the same pattern of frequency hops then diversity is obtained against multipath fading in both the desired signal and interference. However, because of the long-term propagation conditions outlined above, the average $\mathrm{C} / \mathrm{I}$ will vary widely between different mobiles, resulting in poor $90 \%$ and $99 \%$ worst case statistics. If coding across the slots were employed, and different sets of interferers were encountered in successive slots, then a channel code would provide diversity protection against these long-term interference levels, improving the error performance. In addition, there would be less variation in the error performance of the users. In the next section, we outline how to achieve maximum interferer diversity for a given frame length, while in section IV we quantify the performance advantage obtained with such schemes.

\section{Latin squares}

A latin square of order $\mathrm{n}$ is an $\mathrm{n} \times \mathrm{n}$ matrix with entries from a set $\mathrm{R}$ of $\mathrm{n}$ distinct elements, say $\mathrm{R}=\{0,1, \ldots, \mathrm{n}-1\}$ such that each row and column contains every element of $\mathrm{R}$ exactly once. Two Latin squares are said to be orthogonal if the $n^{2}$ ordered pairs (i,j), where $i$ and $j$ are the entries from the same position in the respective squares, exhaust the $n^{2}$ possibilities, i.e., every ordered pair occurs exactly once.

If $\mathrm{n}$ is prime, then there is a simple construction for a family of $\mathrm{n}-1$ mutually orthogonal latin squares. For $\mathrm{a}=1, \ldots, \mathrm{n}-1$ we define an $\mathrm{n} \times \mathrm{n}$ matrix $\{\mathrm{a}\}$ by setting

$$
\{a\}_{i j} \equiv a i+j(\bmod n)
$$

It is easy to verify that each matrix is a latin square: for fixed $i$, the numbers $a i+j, j=0, \ldots, n-1$ are distinct, and for fixed $\mathrm{j}$, the numbers $\mathrm{ai}+\mathrm{j}, \mathrm{i}=0, . ., \mathrm{n}-1$ are distinct, in both cases assuming modulo arithmetic. It remains to verify that if $a \neq b$, then the matrices $\{a\}$ and $\{b\}$ are orthogonal. This amounts to showing that for all $\mathrm{c}, \mathrm{d}=0,1, \ldots, \mathrm{n}-1$, there is a unique solution $(\mathrm{i}, \mathrm{j})$ to the linear system 


$$
\begin{aligned}
& c \equiv a i+j \\
& d \equiv b i+j
\end{aligned}
$$

This follows from the fact that the determinant of this linear system is $a-b$, which is non-zero $(\bmod n)$.

If $\mathrm{n}$ is prime, then the residues modulo $\mathrm{n}$ form the finite field of order $\mathrm{n}$. The above construction extends to finite fields of prime power order in the natural way: now $\mathrm{R}$ is the set of field elements, and for every non-zero field element a, we define a matrix $\{\mathrm{a}\}$ with the $\mathrm{ij}^{\text {th }}$ entry $\{\mathrm{a}\}_{\mathrm{ij}}=$ $\mathrm{ai}+\mathrm{j}$, where addition and multiplication take place in the field.

There is a rich combinatorics literature that relates mutually orthogonal latin squares, projective planes, and planar ternary rings. We refer the read to Hughes and Piper [5] for more information.

\section{Examples}

For $\mathrm{n}=5$, the construction described above produces 4 mutually orthogonal latin squares, as listed in Figure 1. Notice that the matrices $\{2\},\{3\},\{4\}$ can all be obtained from $\{1\}$ by permuting rows. These row permutations are all powers of $\mathrm{P}^{2}: \mathrm{P}_{4}=\mathrm{P}_{2}^{2}$, and $\mathrm{P}_{3}=\mathrm{P}_{2}^{3}$ which follows from $4 \equiv 2^{2}(\bmod 5)$ and $3 \equiv 2^{3}(\bmod 5)$.

For $n=8$, the construction makes use of the finite field $\mathbf{F}_{8}$ which results from appending a root of the primitive polynomial $\mathrm{p}(\mathrm{x})=\mathrm{x}^{3}+\mathrm{x}+1$ to $\{0,1\}$. The elements of the field, and the resulting matrices and listed in Figure 2. Again note that every matrix is obtained from $\{1\}$ by permuting rows using some power of the permutation $\mathrm{P}_{2}$. This will be true in general because every field has a primitive element. In this example, 2 represents $\mathrm{x}$, and the powers of $\mathrm{x}$ exhaust the non-zero field elements.

\section{Implementation}

The above construction may be used in a synchronous cellular radio system. That is, the timing of the slot boundaries must be aligned across cells, with guard bands potentially required for large cells to account for propagation delays from nearby cells. Each cell is assigned a matrix $\{a\}$ in the set of n-1 mutually orthogonal latin squares. Then up to $\mathrm{n}$ users can be accomodated in any 
cell, each user being assigned a field element. For example, suppose $n=8, a=5$, and a user is assigned the element 7 . We might interpret the rows as frequency slots and the columns as time slots for a frequency hopped system. Then at time 0 , frequency 3 is transmitted, at time 1 frequency 6 is transmitted, etc. Except for other cells which also are assigned matrix $\{5\}$ there will be at most one collision with any particular user at any given time/frequency pair over the 8 slot duration of the frame. If a regular re-use pattern of 7 cells is used for the matrices, then the signals from users sharing the same hopping patterns will be heavily attenuated. In this way, each user suffers collisions from an ensemble of users occupying the nearby cells, rather than with one dominant interferer. Obviously, with a larger value of $n$, the re-use distance increases and the randomization of interference hits is more complete. Also, the rows might be reinterpreted as time slots in a frame, and the columns as frame positions within a superframe for a pure TDMA system.

The construction allows each mobile to quickly generate its own time/frequency hopping pattern given minimal information transfer from the base station. The only information required, given that $\mathrm{n}$ and the primitive polynomial $\mathrm{p}(\mathrm{x})$ are known, is the matrix number, a, and the field element, e. Then if $i$ is the row number, the column corresponding to $i$ is just $j \equiv e+a i(\bmod p(x))$. Hence, the overhead involved in communicating the hopping pattern is negligible. Note that we cannot get every possible value of $n$ using this construction. However, primes and prime powers are dense for moderate $\mathrm{n}$, affording a wide range of choices.

A very similar construction has recently been proposed by Wyner [6] using a combinatorial configuration constructed by Roche [7] for use in wideband multi-tone systems, to assure that users in nearby cells interfere in at most one tone.

\section{Coding strategies}

There are several ways in which orthogonal latin squares may be used in a wireless system to improve capacity. The straightforward approach would be to use repetition coding and use some combining strategy such as maximal ratio combining or slot selection. This will take advantage of the averaging of interference levels made possible by the technique. However, it is very wasteful of bandwidth. A more fruitful approach which we outline below is use channel coding in combiniation with interleaving to spread information over multiple slots. With the proper choice of decoding metric, the channel coder then effectively performs diversity combining to find the most likely transmitted sequence. This realizes the diversity gain inherent in the latin squares technique and in addition yields a coding gain. The use of channel coding with the latin squares technique 
results in an interference-limited system. That is, C/I is determined by the fraction of slots occupied, rather than by explicit spatial frequency re-use restrictions. In the following, we outline the consequences of this for channel code selection, and then discuss some of the tradeoffs for channel code design, slot duration, and metric choice for a low-delay system. The section is concluded with two illustrative examples.

\section{Trellis coding alternatives}

In conventional convolutional coding, every bit is encoded and extra symbols are transmitted to accomodate the redundancy introduced by the encoder. If the same energy is allocated to each information bit transmitted, the interference power is not changed in the system. Nevertheless, the usual bandwidth expansion penalty is paid against AWGN, and there is a price in signal to interference ratio, as follows. Consider a rate-1/2 code. An uncoded system with the same throughput, bandwidth, and energy would use half the slots with twice the power, and produce the same interference. Thus, the redundancy introduced in coding degrades the signal to interference ratio by 3 $\mathrm{dB}$ as for additive white noise. However, because there is coding gain, the coded system can actually operate at a much lower power level, or alternatively tolerate an increased level of interference, i.e., allow more users.

In trellis coded modulation the redundancy of the encoder is accomodated in expansion of the signal constellation. Clearly both bandwidth and interference are unaffected by such schemes. However, the distance between signal points in the signal constellation shrinks, resulting in a power penalty against both noise and interference. This penalty is much more severe than that obtained with ordinary convolutional coding, and should be paid only if bandwidth expansion is not possible, as for example in TDMA systems. Define the efficiency $\eta$ as the time-bandwidth product occupied by active mobiles divided by the time-bandwidth product of the system. For the propagation environment outlined in section II, a system with $\eta=1$ would have a signal to interference ratio of $3 \mathrm{~dB}$. Clearly the number of users must be drastically reduced to permit an uncoded system to operate. On the other hand, coded systems can operate at very low signal to noise ratios. Rate $1 / \mathrm{n}$ codes can successfully operate at lower SNR with the same number of states as $\mathrm{n}$ gets larger, although the coding gain against AWGN (which includes the rate-reduction penalty) is to first order independent of $n$ (see [8]). For the same energy per transmitted bit, use of a rate $1 / n$ code improves the signal to interference ratio per bit by the factor of $n$, the same effect as reducing the occupancy in an uncoded system by the factor $n$. However, channel codes provide

gain, and so presuming that there remain open slots, the number of users can actually be increased relative to the uncoded system. This tradeoff is different from bandwidth-limited systems, where 
use of convolutional coding implies a reduction in the number of users, if the frequency re-use is not changed. Thus, convolutional codes provide a more favorable gain/complexity tradeoff in this application than TCM, and increase the number of users permitted to be simulataneously active.

The question is now how to use convolutional codes to obtain coding gain and diversity protection with moderate decoding delay and complexity. To make matters more concrete, we assume that a slow-hopped system employing differential quadrature phase shift keying (DQPSK) is used. The intended application is compressed speech at $8 \mathrm{~kb} / \mathrm{s}$. An uncoded system might be operated at $4 \mathrm{kbaud}$, occupying a bandwidth of $5 \mathrm{kHz}$. The symbol duration is $250 \mu \mathrm{s}$, which is very large compared to either the delay spread or group delay variation of the typical mobile channel. A convolutional code will be used, with interleaving across slots. In $20 \mathrm{~ms}, 80$ symbols are transmitted. To achieve reasonable frequency diversity in $20 \mathrm{~ms}$ and keep the decoding and interleaving delay reasonable, we must hop every few symbols.

For coding to realize the maximum interference and frequency diversity, the signal to interference ratio for successive symbols should be uncorrelated. This implies that coding must be interleaved across slots. For a delay constraint of $20 \mathrm{~ms}$ and slot duration of $\mathrm{m} \mathrm{ms}$, the truncation depth of the Viterbi decoder can be no more than 20/m. Indeed, it can be less if each code branch corre-

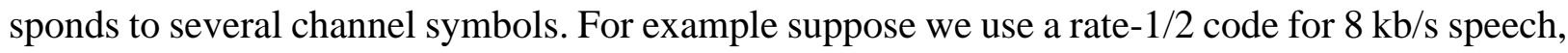
and modulate using DQPSK at 8 kbaud. If slots consist of 8 symbols, then each slot lasts $1 \mathrm{~ms}$ and a truncation depth of 20 is permitted. The full minimum distance of the 32 state code is obtained with a truncation depth of 19 [8], while codes with larger minimum distance will not provide significantly better performance unless the truncation depth is increased. Thus, complexity is limited by delay unless shorter slots are used. An alternative to the rate-1/2 code is the 32 state rate-1/4 code with minimum distance 18 . Keeping 8-symbol slots but now signalling at 16 kbaud, there are a total of 320 symbols in $20 \mathrm{~ms}$, or 40 slots. Each code branch corresponds to symbols in two successive slots. Therefore by decreasing the rate of the code, diversity can be increased. There is little cost in complexity since there are still the same number of states and branches entering each state, and branches are processed at the same speed. Moreover, the truncation depth in branches is a less severe function of minimum distance for rate-1/4 codes [9], so that roughly the same depth vs. number of states tradeoff is obtained. The branch computations are more complicated since demodulation must be accomplished at twice the speed, and there is less flexibility in increasing the number of mobiles when the interference from nearby cells is lighter. Rate-1/2 and rate-1/4 codes will be compared in the simulated examples.

\section{Decoding Metrics}


Any diversity combining device benefits from channel state information, i.e., knowlege of the signal and interference levels. Accurate information is quite difficult to obtain in mobile radio. If short slots are used, the interference estimate will be poor; if longer slots are used, the signal and interference statistics change over the slot duration. We now consider how to estimate the channel state information, and how the quality of the estimates influence the choice of decoder metric. We start with neither a phase reference nor knowlege of the signal power. Signal power may be estimated as the average power over the slot. The mean squared error may be obtained by computing the minimum distance between any pair of received points and the allowable phase transitions in the signal set. Let the set of received channel symbols over the slot be $\{$ ri, $i=1,2, \ldots, N\}$. Then the estimate of the signal power

$$
\hat{\mathrm{S}}=\frac{1}{\mathrm{~N}} \sum_{\mathrm{i}=1}^{\mathrm{N}}\left|\mathrm{r}_{\mathrm{i}}\right|^{2}
$$

Let the set of transmitted signals be $\{x j, j=1, . .4\}$ corresponding to four phases on the unit circle, with $\mathrm{x}_{1}$ corresponding to a phase of 0 . There are four allowable phase transitions in DQPSK between times $i$ and $i+1$. The estimate of the squared error assuming phase transition $\mathrm{j}$ is given by

$$
e_{i j}=\min \left\{\left(r_{n i}-x_{1} e^{j \theta}\right)^{2}+\left(r_{n i+1}-x_{j} e^{j \theta}\right)^{2}\right\}
$$

where $r_{n i}$ is the power-normalized received signal and the minimization is performed over all possible starting phases $\theta$. The squared error estimate $e_{i}$ for the phase transition between times $i$ and $i+1$ is then found by minimizing over the $x_{j}$, so that the estimated mean squared error is given by

$$
\hat{\mathrm{E}}=\frac{1}{\mathrm{~N}-1} \sum_{\mathrm{i}=1}^{\mathrm{N}-1} \mathrm{e}_{\mathrm{i}}
$$

The estimate of $\mathrm{C} / \mathrm{I}$ is then $\hat{\mathrm{S}} / \hat{\mathrm{E}}$. For example, consider a phase transition of 90 degrees. The reference points lie on the unit circle with a phase difference of 90 degrees, but with unknown starting angle. If this were the true transition, then the most likely starting angle would be the one that minimizes the squared distance between the (ordered) received sequences and the reference points. The distances can be tabulated for any combination of quantized and power-normalized received signal points for each allowable transition. The decision is for the transition with the least distance, and the estimate of the squared error is a value from the table. This procedure overestimates $\mathrm{C} / \mathrm{I}$ because if errors actually occur we record the distance to the erroneous rather than the true symbol, and also because the total power includes the interference.

The $\mathrm{C} / \mathrm{I}$ determined for the slot may be used to weight decisions. For example, it could be 
used make a decision for antenna selection diversity or to weight the values for maximal ratio combining. It may also be used with a "soft" decoder metric. The squared distances for each allowable transition can be divided by $\hat{\mathrm{E}}$ for the slot to form the branch metrics for a rate-1/2 code, or part of the branch metric for lower rates. This is not the optimal metric even if the C/I estimate is good; however the maximum likelihood metric requires a set of look-up tables to cover the range of C/I, which is very broad. This metric has the virtue of relative simplicity of implementation and includes both distance and signal to interference ratio information. It is in the spirit of a metric proposed by Ketchum and Wallace [10] for use with Reed-Solomon codes and QPSK, which while also not maximum likelihood, performed quite well in simulations.

We have conducted computer simulations of the 32 -state rate-1/2 and rate-1/4 codes with this soft metric, and hard decision decoding with a slot erasure threshold (based on the estimate of $\mathrm{C} /$ I). We have considered two cases: 1) a benevolent genie supplies perfect $\mathrm{C} / \mathrm{I}$ estimates and 2) $\mathrm{C} / \mathrm{I}$ is estimated as above with a slot duration of 17 symbols. Representative results are presented in Table 1. The simulations assume separate decoders, interleaved across slots. There are twenty slots per frame in the case of rate-1/2 codes, and forty per frame for rate-1/4 codes. Here the interference hopping patterns were randomly generated with the provision that they hit users in cell 0 in only one slot per frame, roughly equivalent to the effect of the constructions of section III. We see that the channel state information is extremely valuable, particularly in attempting to achieve low error rates. With perfect channel state information, soft decisions outperform hard decisions in all cases. However, the estimates obtained in practice are so poor that hard decision decoding with a $4 \mathrm{~dB}$ erasure threshold actually performs better for lower error rates for the rate-1/4 code.

Because the gap in performance between known and estimated C/I is so large we have investigated the possibility of estimating $\mathrm{C} / \mathrm{I}$ from a known training sequence for each slot. Accounting for the overhead involved, the results are worse than for estimating it from the data. It would appear that a very large number of symbols are required to get a useful estimate of the interference power. Consequently, a hard decision metric is a reasonable choice, given the considerable reduction in decoder complexity. However, the relative merits of hard and soft decisions depend on the code, the antenna diversity scheme, and the rate of change of the channel.

\section{Example 1}

In this example, we consider DQPSK modulation. Each user gets 40 slots per frame, each time/frequency slot consisting of one reference symbol and 16 information-bearing symbols. The slots hop in frequency throughout the frame, and we assume that fading is independent in succes- 
sive slots. Coding is across slots, accomplished using a rate-1/4 code, which selects two symbols in successive slots for each branch. Preceding the decoder there may be two-branch antenna selection diversity. It was found that performance was best using the criterion of the largest $\mathrm{C} / \mathrm{I}$ estimate, rather than largest power estimate or combining using either of these criteria. Simulations were conducted over multiple user sets, for a variety of efficiencies. The figure of merit is the fraction of mobiles that experience a bit error rate in excess of $10^{-3}$ for a given use of time and bandwidth.

In Figure 3 we plot the results for the assumption that antenna selection diversity is employed, and no randomization of hopping patterns between cells, labelled as curve 1 . We plot call blocking probability versus number of $8 \mathrm{~kb} / \mathrm{s}$ users per $1.25 \mathrm{MHz}$ bandwidth, to assist in comparing to the CDMA results of [3]. We have removed the gain due to voice activity factor from the latter. All results assume equal loading. Note that $1.25 \mathrm{MHz}$ is not wide enough for the assumption of independence of fading levels to be valid. For example, the CDMA calculations assume only diversity of order 2 for this bandwidth. Our system would have to hop over a wider bandwidth for the independence assumption to be valid, and obviously a direct sequence spread spectrum system could also make use of a wider bandwidth to improve diversity. It is clear in any case that performance of the slow-hopped system is inferior at the design target of $1 \%$ blocking probability.

However, the picture changes when a method such as latin squares is employed. In Figure 3 we also plot results for single and dual antenna branch selection, in curves 2 and 3 respectively. Dramatic gains are evident. Even without antenna diversity, the system gives roughly the same performance as CDMA at the target of $1 \%$ blocking probablity. The system with antenna diversity achieves nearly double the capacity. While it is true that this improvement is achieved assuming a wider hopping bandwidth, in addition our scheme has lower decoding delay and complexity.

Decoding delay in this example is roughly $40 \mathrm{~ms}$. It can be reduced at some cost in efficiency by reducing the number of symbols per slot to 8 . The computer simulation results indicate roughly a $20 \%$ reduction in capacity for $1 \%$ blocking probability.

Also of interest is the increase in the number of users when the surrounding cells are more lightly loaded. In CDMA, the main limiting factor is the interference within the cell, which will cause the error rate to rise with the number of users. However, for a syncrhonous hopped system what limits the number of users in any given cell is the interference they cause in other cells. Consequently, the decision to increase the number of users must come from the network rather than through internal quality of service measurments. For example, if there are no calls in all the sur- 
rounding rings of cells, capacity increases to the limit of $\eta=1$. However, even if the immediate neighbors have reduced loading, the allowable capacity increase depends on the loading of their neighbors, and the relative power we may assign to calls in the various cells. Thus, while this system shares with CDMA the characteristic of "soft" capacity, optimum management of this capacity will be more intricate.

\section{Example 2}

In example 1, it was assumed that Rayleigh fading was independent in successive frequency hops. This presumes that hopping takes place over a very wide bandwidth. We now consider what happens when fading is static and wideband (relative to the hopping bandwidth). This might be the situation for example in a relatively narrowband microcellular system, with communication by pedestrians. Fading would in this case be very slow compared to the signaling interval, affording the receiver time to learn both the signal and interference levels. Hopping might take place in either time or frequency, the only purpose being to randomize the interference levels, to improve code performance.

For such situations, it has been suggested that a simple way to make better use of the diversity antenna of a receiver is to adaptively select which antenna to use for transmission based on occassional measurements of signal strength [14]. Power control is then performed based on the antenna actually selected. Thus, selection diversity may be used in transmitting, with optimal combining used in reception. We assume that power control tracks shadowing but not Rayleigh fading.

When good estimates of signal strength and interference power can be made, soft decision decoding and antenna diversity combining using C/I estimates significantly outperform rival techniques. Simulations were run assuming perfect channel knowlege, and using transmit antenna selection diversity and receive antenna combining. Under these conditions, with the rate-1/4 code and time hopping, all slots could be used in every cell, and still meet a $10^{-3}$ criterion for $1 \%$ outage. The outage probability followed a steep curve. By contrast, for TDMA, the outage probability curve is fairly flat, already achieving $6.5 \%$ outage with full occupancy but declining to $1 \%$ outage only when half the slots were left vacant. Both results indicate higher capacity than in Example 1, reflecting the value of the perfect channel state information, and transmitter selection diversity. It is clear that here as well coding and interleaving when combined with latin squares significantly improves performance over using coding alone. 


\section{Conclusion}

The propagation model outlined in section II is too simple to be useful in predicting outage values in actual systems, and in any case we have made numerous idealizing assumptions (e.g., perfect power control). However, while the relative improvement due to use of schemes similar to the very simple method of latin squares in section III may vary, we still expect dramatic improvement. Regardless of the propagation environment, different interferers will have different power levels. The use of latin squares to govern hopping in either frequency or time decreases the dependence of $\mathrm{C} / \mathrm{I}$ across slots, allowing error correcting codes to realize more of their diversity benefit in synchronous systems.

Systems which combine coding with latin squares are interference-limited rather than bandwidth-limited, in common with CDMA. That is, capacity is limited by the interference generated by adding more users, rather than in the number of slots allocated. This is true whether time-hopping or frequency-hopping or some combination is employed. Thus, any technique which reduces interference or decreases the minimum $\mathrm{C} / \mathrm{I}$ threshold for reliable operation directly increases the capacity (e.g., coding, antenna diversity, sectorization, voice activity). However, frequency hopping would be preferred in practice over time hopping since it affords less delay and provides the possibility of realizing frequency diversity without equalization.

Our results demonstrate that low-rate convolutional codes of very modest complexity may be used to achieve significant gain with low delay. A topic for further research is consideration of other codes. In particular, Reed Solomon codes may be attractive in situations where C/I estimates are poor, negating the soft decision advantage of convolutional codes. A recent study for frequency-hopped FSK indicates that convolutional codes are favored for the correct choice of metric [.], but further research with a variety channel models is required to make a definite conclusion.

We are investigating one further possible use of latin squares in combination with coding. In this study, we have assumed that coding should be used to average the interference. However, time- or frequency-hopped systems may also be used to avoid interference. We are investigating distributed algorithms which dynamically select the M slots with best estimated $\mathrm{C} / \mathrm{I}$ out of the $\mathrm{N}$ slots available in a frame. Thus, for the slots with the worst interference no signal is sent. Preliminary results indicate further improvement in capacity.

\section{Acknowlegment}


We thank J.E. Mazo and N. Seshadri of AT\&T Bell Laboratories for their helpful comments on an earlier version of the manuscript.

\section{References}

[1] B. Gudmundson, J. Sköld, and J.K. Ugland, "A Comparison of CDMA and TDMA Systems,” Proc. 1992 IEEE Vehic. Tech. Conference, Denver, May 11-13, 1992, pp. 732735.

[2] H. Sasaoka, "Block coded 16-QAM/TDMA cellular radio system using cyclical slow frequency hopping," Proc. 1992 IEEE Vehic. Tech. Conference, pp. 405-408.

[3] K.S. Gilhousen et al., "On the Capacity of a Cellular CDMA System," IEEE Trans. Vehic. Tech., vol. 40, May 1991, pp. 303-312.

[4] N.R. Livneh, R. Meridan, M. Ritz, and G. Silbershatz, "Frequency hopping CDMA for digital radio,” Proc. Int'1. Commsphere Symposium, Herzliya, Israel, Dec. 1991.

[5] D.R. Hughes and F.C. Piper. Projective Planes. Springer-Verlag: New York, 1973.

[6] A.D. Wyner, "Multi-tone multiple-access for cellular systems,", AT\&T Bell Laboratories Tech. Memo, BL011217-920811-11TM, Nov. 1991.

[7] J. Roche, "Families of binary matrices having nearly orthogonal rows," AT\&T Bell Laboratories Tech. Memo, BL011217-920812-12TM

[8] S. Lin and D.J. Costello. Error Control Coding: Fundamentals and Applications. PrenticeHall: Englewood Cliffs NJ, 1983.

[9] J.B. Anderson and S. Mohan. Source and Channel Coding: An Algorithmic Approach. Kluwer: Boston, 1991.

[10] J.W. Ketchum and M.S. Wallace, "Reed-Solomon coding and slow frequency hopping for narrowband digital cellular systems," Proc. 1992 IEEE Vehic. Tech. Conference, pp. 225228

[11] N. Seshadri and C-E.W. Sundberg, "Coded modulation with time diversity, unequal error protection, and low delay for the Rayleigh fading channel," Intl. Conference on Universal Personal Comm., Dallas, Sept. 1992.

[12] A.R. Calderbank and N. Seshadri, "Multilevel codes for unequal error protection," submitted, IEEE Trans. Inform. Theory, 1992.

[13] J. Hagenauer, N. Seshadri, and C-E.W. Sundberg, "The performance of rate-compatible punctured convolutional codes for digital mobile radio," IEEE Trans. Comm., vol 38 No. 7, pp. 966-980, July 1990.

[14] R.C. Bernhardt, "User access in portable radio systems in a co-channel interference 
environment," IEEE J. Select. Areas in Comm., vol. 7, No. 1, pp. 49-58, Jan. 1989.

[15] V. Lin and G.J. Pottie, “ 